Jeremy J. Ramsden

\title{
Assessing the toxic risks of the nanotechnology industry
}

ABSTRACT. This article analyses the risks facing the nanotechnology industry due to the toxicity of some nanomaterials. The emphasis is on brevity while being comprehensive in coverage.

Nanotechnology Perceptions 9 (2013) 119-134 